Research Paper

\title{
Cancer survival in patients from a hospital-based cancer registry, China
}

\author{
Jian-Guo Chen ${ }^{1,2}$, Hai-Zhen Chen ${ }^{1}$, Jian Zhu ${ }^{2}$, Yan-Lei Yang ${ }^{3}$, Yong-Hui Zhang2, Pei-Xin Huang ${ }^{3}$, \\ Yong-Sheng Chen ${ }^{2}$, Chao-Yong Zhu' ${ }^{1}$, Li-Ping Yang ${ }^{1}$, Kang Shen ${ }^{1}$, Fu-Lin Qiang ${ }^{1}$, Gao-Ren Wang1 \\ 1. Nantong University Tumor Hospital / Institute, 226361 Nantong, China \\ 2. Qidong Liver Cancer Institute, 226200 Qidong, China \\ 3. Haimen City Center for Disease Control and Prevention, 226100 Haimen, China. \\ $凶$ Corresponding author: Jian-Guo Chen, M.D., M.P.H. Nantong University Tumor Hospital / Institute, 226361 Nantong, China; Qidong Liver Cancer Institute, \\ 226200 Qidong, China. Email: chenjg@ntu.edu.cn \\ (c) Ivyspring International Publisher. This is an open access article distributed under the terms of the Creative Commons Attribution (CC BY-NC) license \\ (https://creativecommons.org/licenses/by-nc/4.0/). See http://ivyspring.com/terms for full terms and conditions.
}

Received: 2017.09.27; Accepted: 2018.01.28; Published: 2018.02.12

\begin{abstract}
Purpose: There are few reports on survival rate analysis from hospital-based cancer registries (HBCR) in China, although the National Center of Cancer Registry of China has launched such an effort with the mission to expand the scope of registration and follow-up. Our study aimed to evaluate survival and outcomes of cancer patients from a HBCR in eastern China.

Methods: Active and passive follow-up methods were used to obtain information on survival status for all patients from Qidong City and Haimen City in the databases of our hospital-based registrations from 2002 to 2014. Censor time for survival was 31 st March, 2016. Survival probability was estimated using the life-table method with SPSS Statistics software, and comparison of significant differences in survival rates was tested by Wilcoxon (Gehan) statistic.

Results: The outcomes of 5010 patients were identified in the follow-up for 5244 cases from Qidong and Haimen, with a follow-up rate of $95.65 \%$, and a rate of lost to follow-up of $4.35 \%$. The 1-, 3-, 5-, and 10 -year observed survival (OS) rate in all-combined cancer sites were $59.80 \%, 37.70 \%, 30.82 \%$, and $22.60 \%$, respectively. The top 10 cancer sites in rank were cancers of lung, esophagus, liver, cervix, stomach, breast, colon-rectum, non-Hodgkin's lymphoma, nasopharynx, and ovary, with 5-year OS rates of $12.63 \%, 19.62 \%, 11.69 \%, 66.61 \%, 21.35 \%, 59.43 \%, 36.36 \%, 37.03 \%, 48.95 \%$ and $36.17 \%$, respectively. Females experienced better survival than males for lung, esophageal, liver, nasopharyngeal and pancreatic cancers $(P<0.05)$, but not for other sites $(P>0.05)$. A significant difference was also found between males and females when all-sites were combined $(P<0.01)$. There are significant differences $(P<0.05)$ between the 2015 patients (from Qidong) and the 3001 patients (from Haimen) with 5-year OS rates of $32.72 \%$ vs $29.57 \%$; no significant differences were found for 5 -year OS rates for individual cancer sites $(P>0.05)$ except for liver $(P=0.0005)$ and ovary $(P=0.0460)$ between the two cities. Younger patients had better prognosis, but significance was only seen in cervical $(P=0.0102)$ and nasopharyngeal $(P=0.0305)$ cancers.

Conclusion: The survival rates of each site or of all sites-combined in this setting are consistent with those elsewhere in China and abroad. Discrepancies in overall survival could be affected by the proportion of sites with or without better prognosis. Hospital-based cancer survival is a better index to evaluate outcomes that reflect the levels of comprehensive treatment and improvement of medical and health services.
\end{abstract}

Key words: Neoplasms; cancer registration (hospital-based); follow-up, survival (life-table method)

\section{Introduction}

Cancer survival rate is an important indicator to assess the prognosis of diagnosed cancer patients.
Patient survival data are usually obtained by long-term follow-up. Previous reports on cancer 
survival rates in China are mainly from two sources. One is from population-based cancer registries (PBCR), obtaining prognosis outcome (survival or death) by continuous or periodical follow-up [1-6]. The second is based on data accumulated from clinical cases (usually in a specific site), describing the surviving status of patients. Clinical survival reports are used to summarize the therapeutic effects of care from clinics or hospitals [7]. PBCR survival can reflect the overall situation of cancer diagnosis and treatment, and even the general situation of health care [4-6]; while survival case series could reflect overall treatment effects in a particular medical department or the use of a certain treatments $[7,8]$. However, reports reflecting comprehensive treatment outcomes of a specific hospital are rare. This is because data from hospital-based cancer registries in China generally lack follow-up of the hospital-based cancer patients [9].

Since 2012, the Affiliated Tumor Hospital of Nantong University has established the hospital-based cancer registration system [10], and has used data starting from the year 2002 for hospitalized-patients with malignancy together with patient follow-up to provide a basis for evaluating the prognosis and survival rates of patients.

\section{Methods}

\section{Cancer registration}

A) Hospital-based cancer registry (HBCR): Based on the "Health Information System, HIS) of Nantong Tumor Hospital, the HBCR was established $[9,10]$. During the period of 2002-2014, a total of 175,901 records with any diseases of hospitalized patients were collected, involving 80,147 cases of patients in Nantong Tumor Hospital. Among them, the total number of cancer-related visits was 156,714 person-times, involving 66,066 cases of hospitalized cancer patients that are included in our HBCR database. Furthermore, 54,921 cancer patients $(83.13 \%$ of the total number of cancer patients treated in the hospital), who were from 8 jurisdictions (3 cities, 2 counties, and 3 districts) of the Nantong Area were included in our follow-up list of cancer patients for study. In this report, 5241 cancer patients were from Qidong City and Haimen City (hereinafter referred to as "Qi-Hai patients"), accounting for $9.54 \%$ of the hospitalized patients in the Nantong Area. Qidong and Haimen are the cities located southeast of Nantong City, with the same dialect, and roughly the same economic development, living habits, and health service conditions. Incidences of cancer at the population level are similar, but as Haimen is closer to the geographical location of the Nantong Tumor
Hospital, the numbers of patients admitted to the hospital were somewhat higher than the numbers of Qidong patients.

B) Population-based cancer registry (PBCR): In the Nantong Area, there are two population-based cancer registries: the Qidong Cancer Registry that covers a population of about 1.12 million, and the Haimen Cancer Registry that covers a population of about 1.10 million. These two cancer registries established their cancer registration systems in 1972 and 1999, respectively. Both have joined the national monitoring programme (the National Cancer Registration Network) of the National Central Cancer Registry of China supported by the Ministry of Health of China [6,11]. Their data on cancer have been used or published in both Chinese and English literature [1,2,4-6]. For these reasons we mainly focus on the patients from these two cities in this report.

\section{Follow up methods}

Active follow up and passive follow up are the common methods employed in population-based cancer registries. The specific forms of follow up include letter, telephone, appointment (outpatient), home visits, community follow-up and network follow-up [9]. Based on our database, and by taking advantage of data from PBCRs in Qidong City and Hamen City, we combined the methods of "active" and "passive" to carry out patient follow-up. First of all, according to their personal information (ID number, phone number and address of patients or their family members) registered during hospitalization, telephone follow-up (active follow-up) was performed in order to obtain information on survival status. About $25 \%$ of the patients (or their family members) were contacted, and their outcomes updated. Because of the change (or error) of telephone numbers, no response (out of home or did not answer the phone), a second round of follow-up for patients who did not provide survival information was carried out, by linking with data from the Qidong and Haimen PBCRs. At the same time, an active follow-up in cooperation with the PBCRs for those patients with recorded information of survival was performed. The "censored" time or deadline of the observation for all survivors was March 31, 2016. The process of patient registration and follow up is shown in figure 1 .

\section{Statistical methods}

A) Variables. Some patients were admitted to the hospital multiple times. In this case, the first admission time to the Nantong Tumor Hospital was considered as the starting date for calculation of the survival rate. The main outcome measure was survival time from the first admission to the end of 


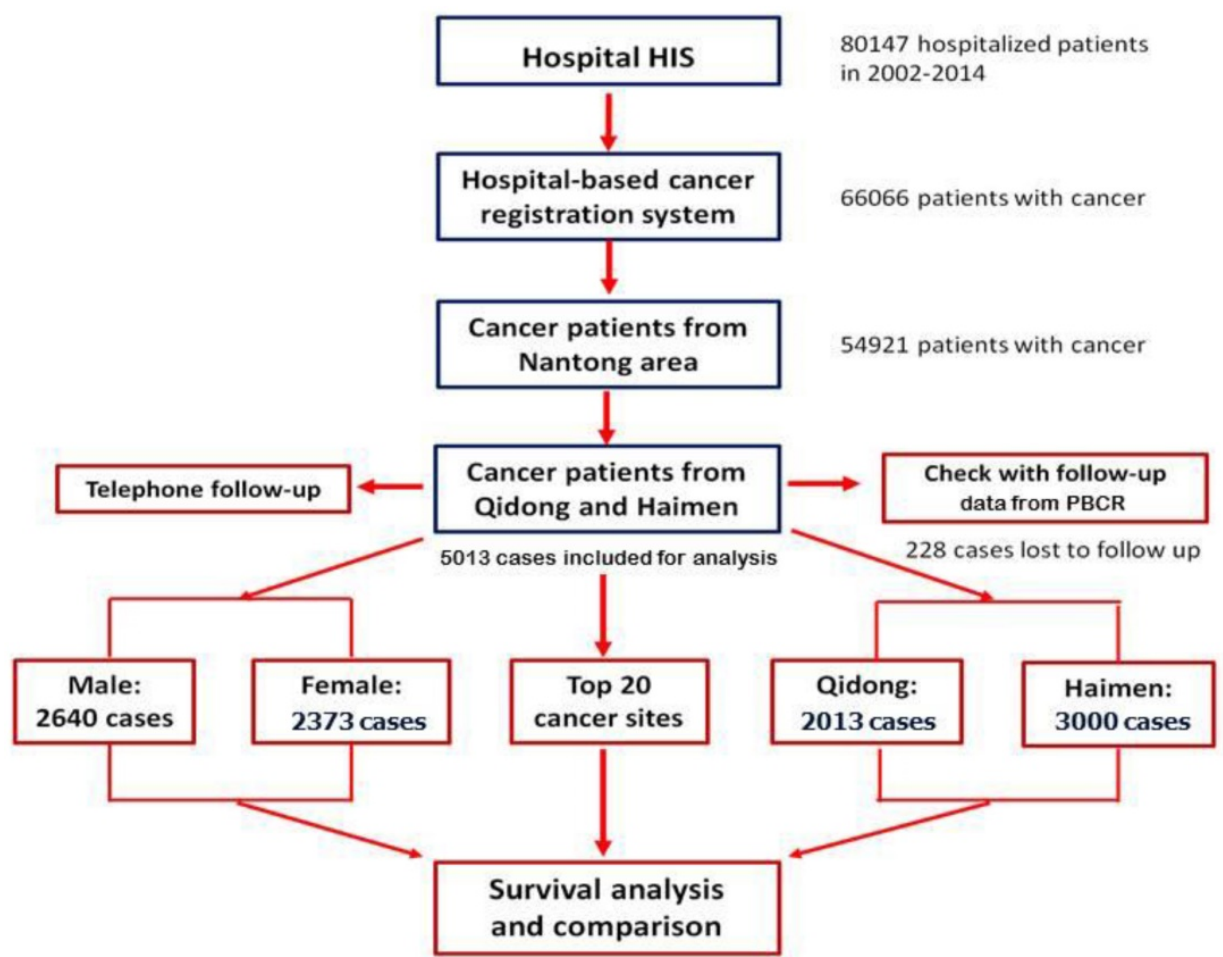

Figure 1. The flow chart of follow up process of Nantong HBCR.

the follow-up period, or to date of death if s/he died before the deadline. The variables concerned in this report are area ( 1 for patients from Qidong, 2 for patients from Haimen), gender ( 1 for male, 2 for female), age group ( 1 for $0-34,2$ for $35-44,3$ for $45-54,4$ for 55-64, 5 for 65-74, and 6 for 75-99), and ICD-10 (according to ICD-10 code digits).

B) Data Analysis. The data were analyzed using IBM SPSS Statistics version 22 software. The total survival rates and the standard errors by ICD-10 site and by the variable area, gender and age group were calculated by the actuarial, or the life-table method, and compared by Wilcoxon (Gehan) statistic. $P<0.05$ was considered as a significant.

\section{Results}

\section{Case distribution}

After active and passive follow up, the survival outcome was traced in 5013 cases (95.65\%), in which 2013 came from Qidong, and 3000 from Haimen. A total of 228 cases were lost to follow up, with a rate of $4.35 \%(228 / 5241)$. The total numbers of cases and the distribution of the top 20 sites of cancer by area, gender and age group are shown in Table 1.

\section{Overall observation survival rate}

The observed survival rate present in this report is the rate calculated from the first date of the patients who were admitted to the Nantong Tumor Hospital to the deadline of the follow up. The 1-, 3-, 5-, and 10 -year survival rates of all patients were $59.80 \%$, $37.70 \%, 30.82 \%$, and $22.60 \%$, respectively. In the top 20 cancer sites, the highest survival rate was seen for corpus uteri: 1-, 3-, 5-, and 10-year survival rates were $92.47 \%, 81.08 \%, 81.08 \%$, and $78.50 \%$, respectively. The worst survival rate was observed in pancreatic cancer, with survival rates of $12.50 \%, 1.39 \%, 1.39 \%$, and $1.39 \%$, respectively (Table 2 ). The survival curves of the top 20 cancer sites are shown in Figure 2.

\section{Comparison of survival rates between patients from Qidong and Haimen}

Because of the differences in survival rates of different cancer sites, the overall survival rate of all cancer combined is dependent on the relative proportion of sites with better survival rates. The 5 -year survival rate of all cancer combined in patients from Qidong (32.72\%) was higher than that in patients from Haimen $(29.57 \%)(P=0.0001)$ (Figure 3A). The proportion of lung, esophagus, and liver cancers that had worse survival rates accounted for poorer overall survival rate in Haimen patients $(39.29 \%)$ than in Qidong patients $(30.87 \%)$. When cancer specific rates are compared there are no statistically significant differences $(P>0.05)$ between these two regions for the top 20 sites with the exception of cancers of the liver and the ovary (Table 3). 
Table 1. Case distribution of cancer inpatients in Nantong Tumor Hospital by gender, age group and area in 2002-2014.

\begin{tabular}{|c|c|c|c|c|c|c|c|c|c|c|c|c|}
\hline \multirow[t]{2}{*}{ ICD-10 } & \multirow[t]{2}{*}{ Site } & \multirow{2}{*}{$\begin{array}{r}\text { Total } \\
\text { No. }\end{array}$} & \multicolumn{2}{|c|}{ Gender } & \multicolumn{2}{|c|}{ Area } & \multicolumn{6}{|c|}{ Age group } \\
\hline & & & Male & Female & Qidong & Haimen & $0-34$ & $35-44$ & $45-54$ & $55-64$ & $65-74$ & 75-99 \\
\hline C34 & Lung & 872 & 607 & 265 & 304 & 568 & 3 & 34 & 121 & 260 & 344 & 110 \\
\hline C15 & Esophagus & 476 & 341 & 135 & 178 & 298 & 2 & 6 & 51 & 140 & 172 & 105 \\
\hline $\mathrm{C} 22$ & Liver & 475 & 379 & 96 & 160 & 315 & 13 & 72 & 165 & 138 & 72 & 15 \\
\hline C53 & Cervix & 435 & 0 & 435 & 194 & 241 & 12 & 101 & 165 & 89 & 41 & 27 \\
\hline C16 & Stomach & 420 & 282 & 138 & 142 & 278 & 5 & 34 & 61 & 126 & 137 & 57 \\
\hline C50 & Breast & 324 & 6 & 318 & 155 & 169 & 11 & 49 & 108 & 100 & 45 & 11 \\
\hline C18-21 & Colon-rectum & 233 & 136 & 97 & 90 & 143 & 2 & 16 & 37 & 87 & 63 & 28 \\
\hline C82-85 & NHL & 198 & 113 & 85 & 92 & 106 & 18 & 15 & 40 & 52 & 57 & 16 \\
\hline C11 & Nasopharynx & 139 & 102 & 37 & 73 & 66 & 8 & 25 & 48 & 36 & 14 & 8 \\
\hline C56 & Ovary & 134 & 0 & 134 & 53 & 81 & 7 & 14 & 42 & 47 & 21 & 3 \\
\hline C54 & Corpus & 93 & 0 & 93 & 51 & 42 & 1 & 6 & 32 & 36 & 16 & 2 \\
\hline C00-14, but 11 & Mouth cavity & 79 & 55 & 24 & 31 & 48 & 1 & 5 & 14 & 22 & 22 & 15 \\
\hline $\mathrm{C} 25$ & Pancreas & 72 & 39 & 33 & 28 & 44 & 0 & 2 & 8 & 25 & 28 & 9 \\
\hline C67 & Bladder & 54 & 44 & 10 & 27 & 27 & 0 & 2 & 3 & 14 & 24 & 11 \\
\hline C73 & Thyroid gland & 50 & 19 & 31 & 28 & 22 & 5 & 5 & 16 & 12 & 8 & 4 \\
\hline C44 & Skin & 48 & 30 & 18 & 21 & 27 & 1 & 4 & 6 & 8 & 9 & 20 \\
\hline C61 & Prostate & 42 & 42 & 0 & 18 & 24 & 0 & 0 & 2 & 9 & 21 & 10 \\
\hline C51,52,55,57,58 & Female genitalia & 39 & 0 & 39 & 18 & 21 & 7 & 0 & 6 & 7 & 11 & 8 \\
\hline $\mathrm{C} 23,24$ & Gallbladder & 36 & 17 & 19 & 12 & 24 & 0 & 3 & 8 & 10 & 11 & 4 \\
\hline C70-72 & Brain and CNS & 36 & 21 & 15 & 20 & 16 & 3 & 2 & 9 & 13 & 6 & 3 \\
\hline C01-97 & All & 5013 & 2640 & 2373 & 2013 & 3000 & 137 & 473 & 1138 & 1446 & 1291 & 528 \\
\hline
\end{tabular}

A.

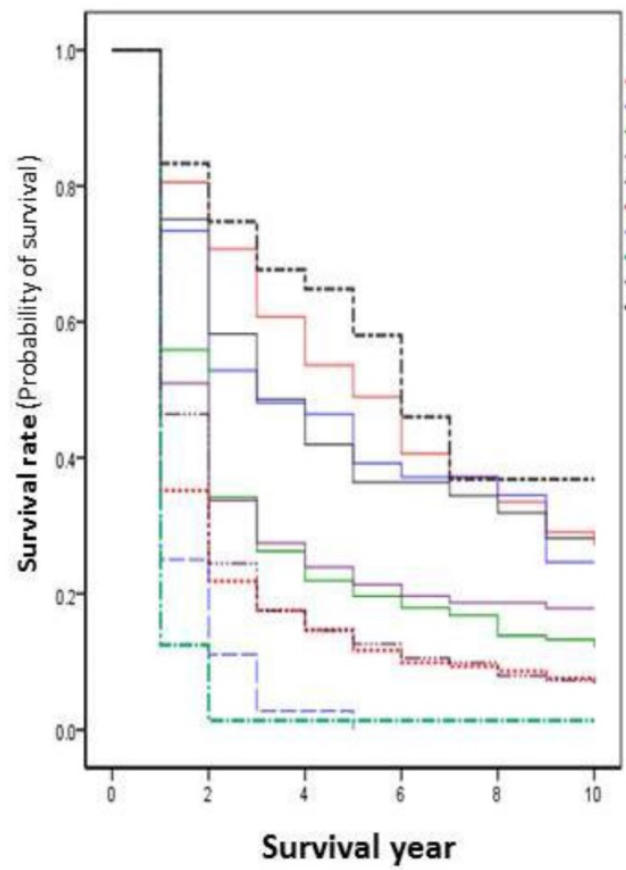

B.

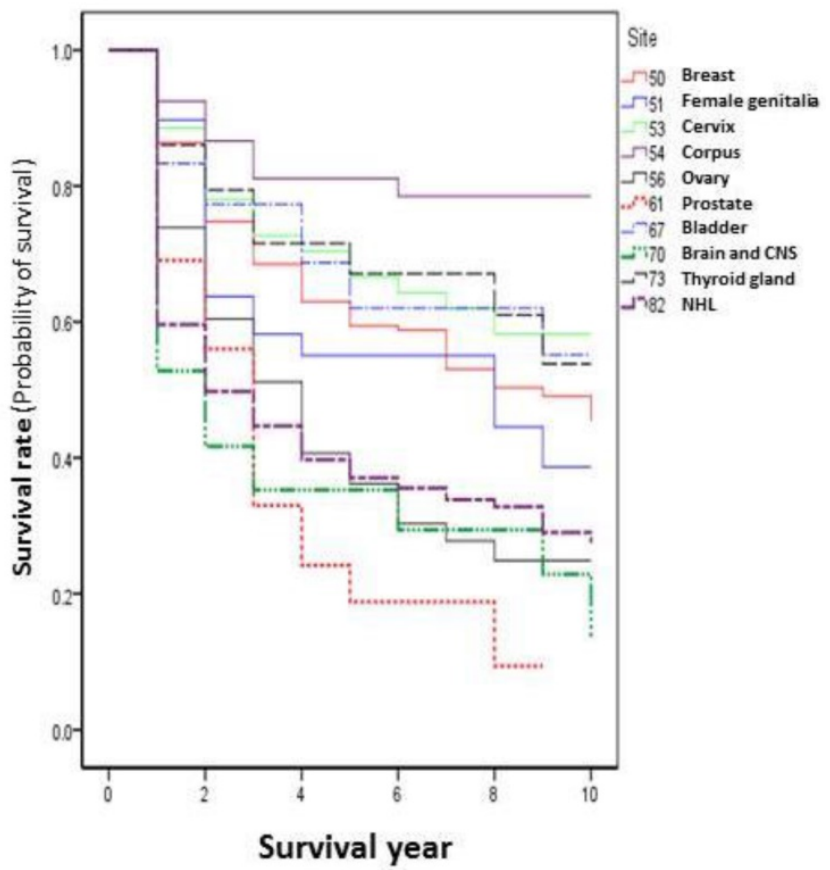

Figure 2. Cancer survival rates by ICD-10 site (Top 20) during 2002-2014.

\section{Gender differences of the survival rates}

The survival rate of all cancers combined was significantly lower in males than in females $(P=0.0000)$ (Figure 3B). Among the top 20 sites, 5 year survival rates of lung cancer, esophageal cancer, liver cancer, pancreatic cancer, and nasopharyngeal carcinoma were significantly higher in females than that in males $(P<0.05)$; while for other sites, there were no significant differences $(P>0.05)$ between women and men (Table 4).

\section{Survival rates by age group}

In general, younger patients with cancer experience relatively higher survival rates than elder patients. This happened as well in our series. The differences become larger after 2 years of survival. The younger the age groups, the higher the survival, 
i.e., the patients of age of $0-34$ had the highest survival rate, those of age over 75 had the worst survival outcome (Figure 4). However, this is not the same when comparisons are made by site, as only the sites of cervix and nasopharynx showed significantly improved survival among younger age groups (Table 5, Figure 4).

Table 2. Survival rates in patients hospitalized in Nantong Tumor Hospital, 2002-2014 (\%).

\begin{tabular}{lrrrrrrrr}
\hline \multirow{2}{*}{ Site } & \multicolumn{2}{c}{ 1-year } & \multicolumn{2}{c}{ 3-year } & \multicolumn{2}{c}{ 5-year } & \multicolumn{3}{c}{ 10-year } \\
\cline { 2 - 10 } & \multicolumn{1}{c}{ SE } & $\%$ & $S E$ & $\%$ & $S E$ & $\%$ & $S E$ \\
\hline Lung & 46.44 & 1.69 & 17.47 & 1.35 & 12.63 & 1.24 & 6.92 & 1.15 \\
Esophagus & 55.88 & 2.28 & 26.24 & 2.06 & 19.62 & 1.92 & 12.14 & 1.87 \\
Liver & 35.16 & 2.19 & 17.52 & 1.80 & 11.69 & 1.68 & 6.34 & 1.86 \\
Cervix & 88.51 & 1.53 & 72.70 & 2.24 & 66.61 & 2.58 & 58.18 & 3.32 \\
Stomach & 50.95 & 2.44 & 27.46 & 2.25 & 21.35 & 2.17 & 17.84 & 2.21 \\
Breast & 86.42 & 1.90 & 68.52 & 2.67 & 59.43 & 3.00 & 45.37 & 4.21 \\
Colon-rectum & 75.11 & 2.83 & 48.60 & 3.39 & 36.36 & 3.52 & 28.14 & 4.14 \\
NHL & 59.60 & 3.49 & 44.69 & 3.58 & 37.03 & 3.59 & 27.44 & 3.93 \\
Nasopharynx & 80.58 & 3.36 & 60.75 & 4.28 & 48.95 & 4.54 & 27.05 & 4.77 \\
Ovary & 73.88 & 3.79 & 51.15 & 4.52 & 36.17 & 4.89 & 24.85 & 5.48 \\
Corpus & 92.47 & 2.74 & 81.08 & 4.31 & 81.08 & 4.31 & 78.50 & 4.88 \\
Mouth cavity & 73.42 & 4.97 & 48.10 & 5.85 & 39.16 & 5.98 & 24.64 & 6.50 \\
Pancreas & 12.50 & 3.90 & 1.39 & 1.38 & 1.39 & 1.38 & 1.39 & 1.38 \\
Bladder & 83.33 & 5.07 & 77.31 & 5.78 & 62.01 & 7.72 & 55.12 & 9.45 \\
Thyroid gland & 86.00 & 4.91 & 71.58 & 6.78 & 67.10 & 7.69 & 53.83 & 10.48 \\
Skin & 83.33 & 5.38 & 67.66 & 6.91 & 58.02 & 7.88 & 36.81 & 9.12 \\
Prostate & 69.05 & 7.13 & 32.95 & 8.11 & 18.80 & 7.81 & 9.40 & 7.71 \\
Female genitalia & 89.74 & 4.86 & 58.19 & 8.00 & 55.04 & 8.16 & 38.62 & 9.84 \\
Gallbladder & 25.00 & 7.22 & 2.78 & 2.74 & 0.00 & 0.00 & 0.00 & 0.00 \\
Brain and CNS & 52.78 & 8.32 & 35.26 & 8.11 & 35.26 & 8.11 & 13.71 & 8.85 \\
All & 59.80 & 0.69 & 37.70 & 0.71 & 30.82 & 0.71 & 22.60 & 0.77 \\
\hline SE = standard error & & & & & & & & \\
& & & & & & & &
\end{tabular}

Table 3. Comparison of 5-year survival rates between patients from Qidong and Haimen, 2002-2014.

\begin{tabular}{|c|c|c|c|c|c|c|}
\hline \multirow[t]{2}{*}{ Site } & \multirow{2}{*}{$\frac{\text { Qidong }}{5-\mathrm{yr}(\%)}$} & \multicolumn{3}{|c|}{ Haimen } & \multirow{2}{*}{$\begin{array}{r}\text { Wilcoxon } \\
\text { Statistic }\end{array}$} & \multirow[t]{2}{*}{$P$ value } \\
\hline & & $S E$ & $5-\mathrm{yr}(\%)$ & $S E$ & & \\
\hline Lung & 12.14 & 2.07 & 12.99 & 1.56 & 3.7608 & 0.0525 \\
\hline Esophagus & 17.31 & 2.95 & 21.00 & 2.51 & 2.7428 & 0.0977 \\
\hline Liver & 14.62 & 3.17 & 10.31 & 1.94 & 12.1354 & 0.0005 \\
\hline Cervix & 65.64 & 3.98 & 67.43 & 3.37 & 0.0065 & 0.9358 \\
\hline Stomach & 20.92 & 3.83 & 21.48 & 2.63 & 0.1584 & 0.6907 \\
\hline Breast & 63.23 & 4.15 & 55.76 & 4.32 & 1.4618 & 0.2266 \\
\hline Colon-rectum & 28.37 & 5.31 & 41.73 & 4.60 & 2.1508 & 0.1425 \\
\hline NHL & 38.91 & 5.30 & 35.45 & 4.87 & 1.4958 & 0.2213 \\
\hline Nasopharynx & 49.16 & 6.13 & 48.50 & 6.75 & 0.5623 & 0.4534 \\
\hline Ovary & 32.40 & 7.64 & 38.77 & 6.33 & 3.9829 & 0.0460 \\
\hline Corpus & 76.43 & 6.28 & 86.67 & 5.62 & 1.8090 & 0.1786 \\
\hline Mouth cavity & 42.95 & 9.70 & 36.69 & 7.60 & 0.1879 & 0.6647 \\
\hline Pancreas & 0.00 & 0.00 & 2.27 & 2.25 & 0.1039 & 0.7472 \\
\hline Bladder & 62.16 & 10.38 & 61.43 & 11.59 & 0.1149 & 0.7346 \\
\hline Thyroid gland & 63.37 & 10.38 & 72.73 & 10.59 & 0.8760 & 0.3493 \\
\hline Skin & 64.13 & 11.09 & 53.64 & 10.80 & 0.0024 & 0.9609 \\
\hline Prostate & 13.80 & 9.53 & 24.00 & 11.77 & 0.8009 & 0.3708 \\
\hline Female genitalia & 70.83 & 11.04 & 42.02 & 10.96 & 3.5508 & 0.0595 \\
\hline Gallbladder & 0.00 & 0.00 & 0.00 & 0.00 & 0.7830 & 0.3762 \\
\hline Brain and CNS & 39.38 & 11.06 & 30.00 & 11.78 & 0.0146 & 0.9038 \\
\hline All & 32.72 & 1.13 & 29.57 & 0.90 & 15.4573 & 0.0001 \\
\hline
\end{tabular}

\section{Discussion}

Since 2002, China has begun to establish their national cancer registration system; furthermore, the importance of cancer registration and follow up of the cancer patients has been emphasized in January 2015 by The National Health and Family Planning Commission of China [12]. This mandate allows for the establishment of the national cancer registration system, to strengthen the standardized management of cancer registration, to provide a nationwide organizational guarantee to enumerate the epidemic status and cancer burden. As of 2016, there are about 347 population-based cancer registries (PBCRs) in China responsible for the work of cancer registration [1].

But so far, only data from 72 of the cancer registries (including the Qidong and Haimen PBCRs) have been included in the national analysis [1]. The PBCR data from Qidong was also included in the compilation of the "Cancer Incidence in Five Continents" published by the International Agency for Research on Cancer [13], and the survival of all sites of cancer between 1972 and 2011 has been reported [6]. In 2006, a first "National Hospital-based Cancer Registration Work Conference", sponsored by the National Center of Cancer Registry, was held in China, with the mission to expand the scope of work in registration and follow-up. However, there are few reports from HBCRs for survival rate analysis [7], except for survival analysis from PBCRs [5,6,14,15]. This is mainly because most of the tumor hospitals have not yet established their HBCR systems. Moreover, even in the established PBCRs, follow up has not been easy to carry out, and the rate of loss to follow up has been high. For instance, in a report from provincial tumor hospitals in China, the follow-up rate of cancer patients was between $65 \%$ and $71 \%$ [16]; thus, it is difficult to obtain complete information of cancer patient outcomes.

Nantong Tumor Hospital, located in the north shore of the Yangtze River, which is the only 3-A Grade Tumor Hospital in Northern Jiangsu Province, has 1200 beds and more than 1207 medical staff. After establishing the hospital based cancer registration system in 2012, it has collected and improved the information of patients with cancer in 2002-2014, and at the same time active follow-up of cancer patients was initiated $[9,10]$. The Qidong Cancer Registry and Haimen Cancer Registry are the two well-run PBCRs in this area. Taking advantage of their long-term databases and their follow-up systems, the combined active and passive methods were used for the case check-up and follow-up in our case series $[6,9,14]$. These measures enabled the follow up rates of the hospitalized patients from Qidong and Haimen in our series to reach $95.65 \%$, thus, the accuracy of the information obtained is greatly improved. 


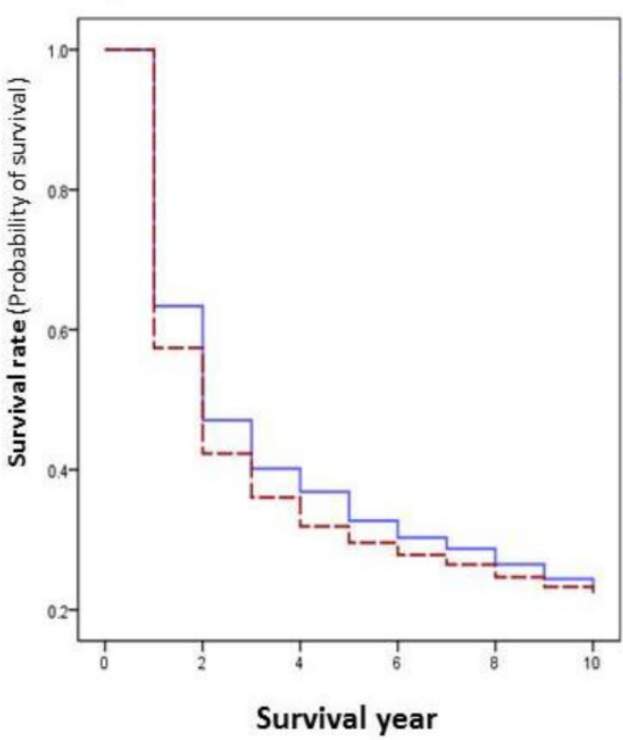

B.

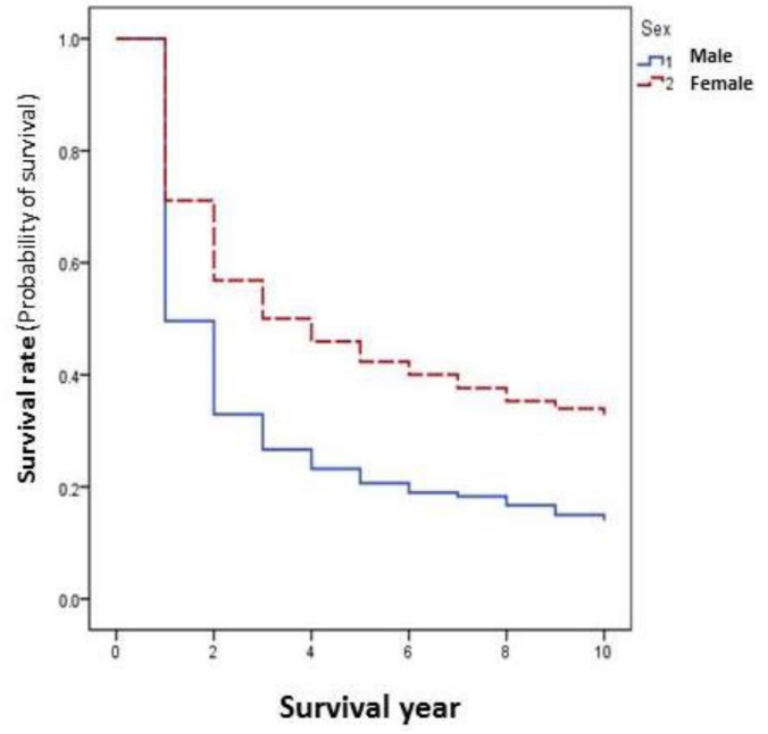

Figure 3. Cancer survival rates by area and by gender during 2002-2014.

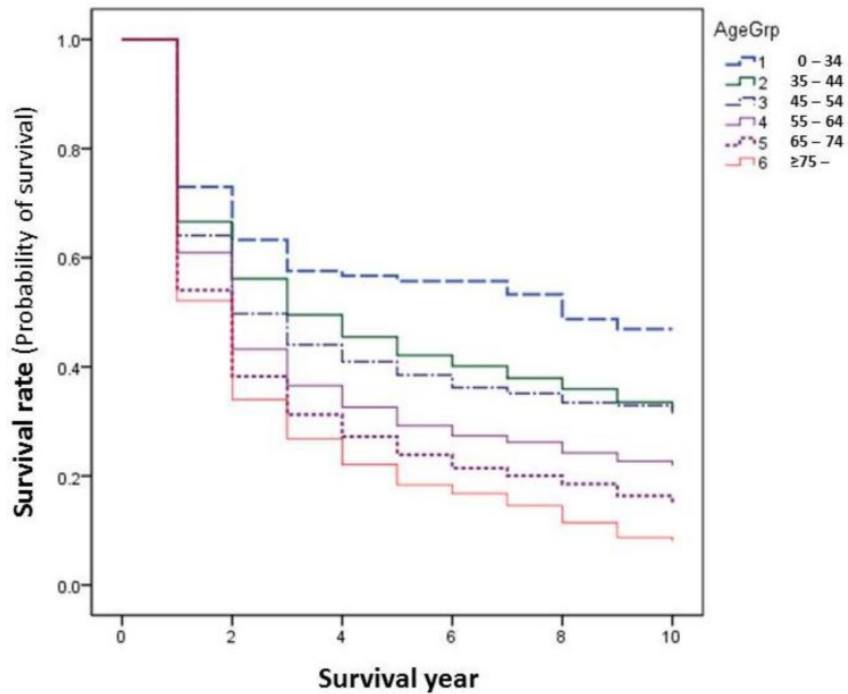

Figure 4. Cancer survival rates by age group during 2002-2014.

In 2015, Zeng HM et al reported that the 5-year relative survival rate of cancer patients from 17 PBCRs in China was 30.9\% (39.5\% in urban areas, and $21.8 \%$ in rural areas) [4]. In this series, the 1-, 5-, and 10-years survival rates for all combined cancer sites were $59.80 \%, 30.82 \%$, and $22.60 \%$, respectively, being close to the average level of the national data. However, the starting point of survival rate for the patients from any PBCR is the first date when he or she is diagnosed as cancer, while the starting point of survival rate for the patients from any HBCR is the first hospitalized date from diagnosis of a cancer to be hospitalized at any hospital. There could be a length (it could be a few days or months or even longer) that has been "cut off", if the patient's first diagnosis due to cancer has been already made elsewhere. In addition, the relative survival rate (RS) is a ratio of the observed survival rate to the expected rate for a group of people in the general population similar to the patient group with respect to gender, age and calendar period of observation; the expected rate is the survival probability of a cohort (hence it is always less than 1 ). Therefore, the RS rate is always greater than the observed survival rate (OS) $[17,18]$ and should be considered in the assessment.

Table 4. The 5-year survival rate (\%) by gender in 2002-2014.

\begin{tabular}{lrrrrrr}
\hline Site & \multicolumn{1}{l}{ Male } & \multicolumn{2}{c}{ Female } & & $P$ \\
& \multicolumn{1}{c}{$\%$} & $S E$ & $\%$ & $S E$ & Wilcoxon & value \\
& & & & & Statistic & \\
\hline Lung & 12.88 & 1.84 & 13.46 & 2.92 & 4.2034 & 0.0403 \\
Esophagus & 18.67 & 2.89 & 26.42 & 4.88 & 10.3556 & 0.0013 \\
Liver & 8.36 & 1.95 & 19.11 & 5.98 & 11.0288 & 0.0009 \\
Cervix & - & - & 67.43 & 3.37 & - & \\
Stomach & 18.80 & 3.08 & 26.89 & 4.89 & 0.0720 & 0.7885 \\
Breast & 0.00 & 0.00 & 56.91 & 4.35 & 8.0626 & 0.0045 \\
Colon-rectum & 39.94 & 5.93 & 44.65 & 7.20 & 0.9074 & 0.3408 \\
NHL & 37.56 & 6.12 & 30.14 & 8.19 & 0.9656 & 0.3258 \\
Nasopharynx & 47.18 & 7.95 & 51.84 & 12.60 & 4.8359 & 0.0279 \\
Ovary & - & - & 38.77 & 6.33 & - & \\
Corpus & - & - & 86.67 & 5.62 & - & \\
Mouth cavity & 33.20 & 8.39 & 44.74 & 16.48 & 1.9705 & 0.1604 \\
Pancreas & 0.00 & 0.00 & 5.00 & 4.87 & 4.2425 & 0.0394 \\
Bladder & 53.13 & 12.86 & 100.00 & 0.00 & 0.1658 & 0.6839 \\
Thyroid gland & 75.00 & 15.31 & 71.43 & 14.37 & 1.2769 & 0.2585 \\
Skin & 53.67 & 13.15 & 60.00 & 15.49 & 1.2769 & 0.2585 \\
Prostate & 24.00 & 11.77 & - & - & - & \\
Female genitalia & - & - & 42.02 & 10.96 & - & \\
Gallbladder & 0.00 & 0.00 & 0.00 & 0.00 & 0.4193 & 0.5173 \\
Brain and CNS & 28.57 & 17.07 & 29.63 & 16.38 & 0.9835 & 0.3213 \\
All & 20.65 & 0.84 & 42.34 & 1.11 & 332.5842 & 0.0000 \\
\hline & & & & & &
\end{tabular}


Table 5. Comparison of survival in age groups by site.

\begin{tabular}{|c|c|c|c|c|c|c|c|c|c|c|c|c|c|c|}
\hline & \multicolumn{2}{|c|}{$\underline{0-34}$} & \multicolumn{2}{|c|}{$\underline{35-44}$} & \multicolumn{2}{|c|}{$\underline{45-54}$} & \multicolumn{2}{|c|}{$\underline{55-64}$} & \multicolumn{2}{|c|}{$\underline{65-74}$} & \multicolumn{2}{|c|}{$\underline{75-99}$} & \multirow{2}{*}{$\begin{array}{r}\text { Wilcoxon } \\
\text { Statistic }\end{array}$} & \multirow[t]{2}{*}{$P$ value } \\
\hline & $\%$ & $S E$ & $\%$ & $S E$ & $\%$ & $S E$ & $\overline{\%}$ & $S E$ & $\overline{\%}$ & $S E$ & $\%$ & $S E$ & & \\
\hline Lung & 33.33 & 27.22 & 10.13 & 5.69 & 13.07 & 3.21 & 14.89 & 2.37 & 11.62 & 1.97 & 10.03 & 3.33 & 5.5606 & 0.3514 \\
\hline Esophagus & 50.00 & 35.36 & 23.81 & 19.89 & 30.42 & 6.55 & 18.82 & 3.37 & 22.63 & 3.54 & 9.02 & 2.99 & 10.3085 & 0.0670 \\
\hline Liver & 23.08 & 11.69 & 6.57 & 3.63 & 12.48 & 2.77 & 8.46 & 2.84 & 19.01 & 5.32 & 0.00 & 0.00 & 9.8272 & 0.0803 \\
\hline Cervix & 81.48 & 11.94 & 70.92 & 5.35 & 72.57 & 3.77 & 66.70 & 5.82 & 43.78 & 9.67 & 44.64 & 10.12 & 15.0381 & 0.0102 \\
\hline Stomach & - & - & 31.82 & 8.05 & 16.73 & 5.55 & 27.43 & 4.33 & 17.47 & 3.57 & 15.62 & 4.97 & 6.9108 & 0.2274 \\
\hline Breast & 90.91 & 8.67 & 57.84 & 7.51 & 65.53 & 4.88 & 57.96 & 5.57 & 49.73 & 8.29 & 29.29 & 16.78 & 5.5220 & 0.3555 \\
\hline Colon-rectum & - & - & 8.25 & 10.75 & 31.53 & 8.09 & 43.17 & 5.98 & 41.66 & 6.78 & 24.31 & 9.06 & 5.7402 & 0.3323 \\
\hline NHL & 30.76 & 11.37 & 53.33 & 12.88 & 41.26 & 7.96 & 42.29 & 7.12 & 26.35 & 6.18 & 42.86 & 12.59 & 3.7237 & 0.5898 \\
\hline Nasopharynx & 75.00 & 15.31 & 65.66 & 9.91 & 50.31 & 7.68 & 38.35 & 9.03 & 48.22 & 15.32 & 0.00 & 0.00 & 12.3299 & 0.0305 \\
\hline Ovary & 71.43 & 17.07 & 24.49 & 13.11 & 33.40 & 9.11 & 42.40 & 8.19 & 25.19 & 11.17 & - & - & 4.2169 & 0.5186 \\
\hline Corpus & 0.00 & 0.00 & 66.67 & 19.25 & 83.68 & 6.69 & 83.36 & 6.94 & 86.81 & 8.72 & 50.00 & 35.36 & 5.6714 & 0.3395 \\
\hline Mouth cavity & 100.00 & 0.00 & 80.00 & 17.89 & 16.29 & 10.44 & 28.93 & 11.46 & 52.88 & 10.98 & 38.41 & 12.79 & 4.2668 & 0.5117 \\
\hline Pancreas & - & - & - & - & - & - & 4.00 & 3.92 & 0.00 & 0.00 & 0.00 & 0.00 & 5.2383 & 0.2637 \\
\hline Bladder & - & - & 100.00 & 0.00 & 100.00 & 0.00 & 61.22 & 14.01 & 53.57 & 12.56 & 59.23 & 18.97 & 4.1402 & 0.3874 \\
\hline Thyroid gland & 100.00 & 0.00 & 80.00 & 17.89 & 74.18 & 13.47 & 62.86 & 14.93 & 37.50 & 17.12 & 75.00 & 21.65 & 10.1215 & 0.0719 \\
\hline Skin & 100.00 & 0.00 & 100.00 & 0.00 & 83.33 & 15.21 & 75.00 & 15.31 & 77.04 & 14.29 & 28.91 & 11.30 & 8.3778 & 0.1366 \\
\hline Prostate & - & - & - & - & 0.00 & 0.00 & 64.81 & 16.53 & 14.77 & 9.87 & 8.08 & 10.51 & 2.7883 & 0.4254 \\
\hline Female genitalia & 85.71 & 13.23 & - & - & 66.67 & 19.25 & 28.57 & 17.07 & 53.85 & 15.22 & 46.02 & 18.72 & 4.2914 & 0.3680 \\
\hline Gallbladder & - & - & - & - & 0.00 & 0.00 & 0.00 & 0.00 & 0.00 & 0.00 & - & - & 8.0989 & 0.0880 \\
\hline Brain and CNS & 66.67 & 27.22 & - & - & 44.44 & 16.56 & 29.91 & 12.92 & 33.33 & 19.25 & - & - & 4.2914 & 0.3680 \\
\hline All & 41.54 & 6.11 & 28.88 & 3.31 & 25.34 & 1.93 & 18.74 & 1.61 & 11.86 & 1.49 & 14.51 & 2.54 & 114.8121 & 0.0000 \\
\hline
\end{tabular}

From our 5-year survival rates of cancer by site, the prognosis of corpus cancer $(81.08 \%)$, thyroid cancer $(67.1 \%)$ and cervical cancer $(66.61 \%)$ were the best, compared with others in top 20 sites, followed by bladder cancer (62.01\%), and breast cancer (59.43). The worst prognoses could be seen in gallbladder cancer and pancreatic cancer as their 5-year survival rates were $0 \%$ and $1.39 \%$. Prognosis is poor for the sites of liver, lung, prostate and esophagus, with rates of $11.69 \%, 12.63 \%, 18.80 \%$, and $19.62 \%$, respectively. These results of survival rank were roughly similar to other published data. In the United States during 2005-2011, Miller KD et al reported that the 5-year RS rates were higher for thyroid cancer (98\%), breast cancer $(89 \%)$, corpus cancer $(82 \%)$, and cervical cancer (68\%), but lower in pancreatic cancer $(7 \%)$, liver cancer $(17 \%)$, lung cancer $(17 \%)$, and esophageal cancer (18\%) [19]. In Asian areas, the 5-year survival rates, were $45.4 \%$ - $81.2 \%$ for cervical cancer and $58.6 \%-88.1 \%$ for female breast cancer [20]. A report from India showed that the 5-year survival rates were lower for pancreatic cancer (4.1\%), lung cancer $(7.0 \%)$, gastric cancer $(10.1 \%)$, and esophageal cancer (11.8) [21]. A report from PBCRs showed that the 5-year relative survival rates of lung cancer, esophageal cancer, liver cancer, gastric cancer and breast cancer in rural areas of China were $11.2 \%, 21.2 \%, 6.3 \%, 24.9 \%$ and $55.9 \%$, respectively [4]. In this report, our data shows that the 5-year observed survival rates of cancer for above mentioned sites were $12.63 \%$, $19.62 \%, 11.69 \%, 21.35 \%$ and $59.43 \%$, respectively. Among these the 5-year OS rate of liver cancer, for instance, was obviously higher than that of the national average level in China.
It can be seen from this report that the prognosis of common cancers in hospitalized patients from the Qidong and Haimen area, is equivalent to the national results and has no significant differences compared, site by site, with data from other areas. But, the 5-year survival rate for all cancers combined was only $30.82 \%$, which is far lower than that in Western countries: such as $67 \%$ in the United States [19]. One of the most important reasons is that the major sites of cancer, for example, breast cancer, prostate cancer in these countries, have available screening efforts, better prognoses and account for a relatively large proportion of their overall cancer burden. As their 5 -year RS rate reaches to $89 \%$ and $99 \%$, respectively [19], and results in better prognosis for all sites combined. Among all the cancer patients involved in this report, the proportion of sites which had a worse prognosis (e.g., lung, liver, esophagus and stomach) accounted for $44.74 \%(2243 / 5013)$. Thus the overall OS rate of all cancers is certainly affected by the proportional weight of the main cancer sites.

In general, the OS rate for cancers combined is higher in women than in men. Except for the gender-specific cancer sites, the observed survival rate in women is always higher than that in men. In our results, the significant differences $(P<0.05)$ between genders were found in esophageal cancer, liver cancer, nasopharyngeal cancer and pancreatic cancer. The comparisons of survival rate by site between patients from Qidong and Haimen showed no significant differences $\left(P_{S}>0.05\right)$, except for the sites of liver and ovary $\left(P_{S}<0.05\right)$. For the survival rate of all cancer combined, the analysis showed that the 5-year survival rate in patients from Qidong 
$(32.72 \%)$ was higher than that in patients from Haimen $(29.57 \%)$ because the proportion of cancer sites that had poor survival such as lung, esophagus, liver and stomach was higher in Haimen patients $(48.63 \%)$ than in Qidong patients $(38.95 \%)$, so they "pull down" the overall survival rate of cancer combined in those from Haimen patients. It reminds us that in comparison with the overall survival rate of cancer patients in various areas, the weights of the proportion of patients with different sites of cancer that had different prognosis should be taken into consideration.

Even for a special cancer site, the proportion of early stage is a key factor of overall survival prognosis. In a report from Singapore-Malaysia Breast Cancer Registry (SMBCR), two hospital-based breast cancer databases were merged into a study to analyze the clinical and pathological tumor characteristics, treatment patterns, and outcomes of breast cancer between 1990 and 2007. Their findings show that the survival rate was related with early TNM stage. The 5-year survival rates were 95.6\%, 93.6\%, 76.0\%, 44.1\%, and $9.3 \%$ at stage from 0 to IV, respectively, but, simply reflect that the weight (proportion) of early stage 0 and $I$ is small (stage 0 and I accounted for $28 \%$, stage III and IV accounted for $25 \%$ ), and thus the overall survival was relatively low, being $67.6 \%$ [22].

At present the major cancer sites in China are lung, stomach, liver, and esophagus; cancers of the liver, and the lung have relative poor prognosis [4]. Obviously if the proportion of these two cancer sites are much higher than in other areas, the overall survival rate of cancer must be affected (reduced). Therefore, when comparing cancer survival rates in different regions, the "standardization" methods (considering the weights of different cancer sites), or the comparison by site should be kept in mind.

Age is an important factor that would affect the prognosis of cancer. When all sites were combined, the outcomes showed a trend that survival decreased with age. However, this is not always true for each site. In this report significant trends were observed only for cancers of cervix and nasopharynx, but not the other sites. Better survival could be seen in the "middle" age group for some sites, for instance, at age group of 55-64 for colon-rectum cancer, age group of 35-44 for NHL, and 65-74 for the site of corpus.

In our previous study [9], we found that the source of patients in the Nantong Tumour Hospital was related to the geographical location of the surrounding counties and cities, and to their levels of health services. Compared to other counties and cities of the Nantong Jurisdiction Area, the proportion of outpatient and inpatients from the Qidong and Haimen areas is relatively lower, likely resulting from geographic proximity to Shanghai City and is covered by the "radiation range" from Shanghai medical services. In addition, the proportion of various cancer patients from any hospital cancer registry, is not necessarily consistent with the actual level of cancer incidence in the local population. For example, the leading 5 cancer sites were lung, liver, stomach, female breast and colorectal in Qidong in 2011 [2], while the top 5 sites of cancer in patients from Qidong who were hospitalized in Nantong Tumor Hospital were lung, cervix, esophagus, liver and female breast, which mainly depends on the hospital relevant medical disciplines, and their attraction to the patients in local or other surrounding areas. The other example is leukemia, which is about the $8^{\text {th }}$ most common malignancy in Qidong area [2], but these patients were rarely hospitalized in the Nantong Tumor Hospital, with a total number of 10 cases (half from Qidong, other half from Haimen) during the years 2002-2014. Hence, this site is ranked out of the top 20 for all sites.

Therefore, the proportion of each site to the total cancer sites in a hospital is mainly reflected of the characteristics of its medical specialties and the capabilities of the medical services of the hospital, rather than the catchment size of cancer patients or incident level in the location. Cancer survival or prognosis in HBCRs is the reflection of the hospital's comprehensive cancer treatment service capacity and their effects on the patients' outcome. A complete HBCR system and the medical follow-up system are the key of increasing follow-up rate of inpatients / discharged patients, and also is warranted for the evaluation of survival outcome of patients.

There also have some survival analysis from HBCRs, some of them mainly focus on the survival by site/subsite, some on the survival by stage, or by therapy. Fan et al reported the survival of colorectal cancer registered from 1960s to 2008, according to the TNM classification or subtype, showing that the 5-year survival rate increased in ascending, descending, and sigmoid colon cancer and rectal cancer patients; however, not in the transverse colon cancer patients from the 1960s to 2000s [23]. From a Taiwan study, 129 patients with multiple primary malignancies (MPMs) of the digestive system were reviewed and analyzed from a series of 9807 pathologically-proven-cancer patients treated at the Mackay Memorial Hospital from 1991 to 2000. This is, in fact, from the view of analysis, a small clinic case series report referring to cancer survival, but is a "big" series of MPMs referring to cancer diagnosis or classification [24].

The combination use of data from HBCR with PBCR is of benefit for the completeness of follow-up 
and is helpful for obtaining the exact vital status of the study patients and abundant clinical information as well. For instance, in a recent multi-center, hospital-based study on breast cancer patient survival from Beijing, both active and passive follow-up systems were performed coordinately. Hence, the survival status (by stage) of cancer patients were identified, and the personal and clinical or medical information / characteristics (such as histological grade /type, stage at diagnosis, estrogen receptor (ER) status, and Molecular subtype) were abstracted [25].

In summary, the HBCR database will provide an infrastructure for future clinical and health services research and will support intervention for cancer patients on return-to-work and quality of life, such as seen in studies in Japan and in Netherlands [26,27]. In our practice the follow-up rate, and the accuracy of diagnosis of outcome data has effectively improved through the combined methods of "active follow-up" and "passive follow-up". However, there may be limitations for use of data from any HBCR that provides information reflecting the specialty features of the department or hospital, but not the epidemic trend of the cancer site in the local population. We believe that this study helps to provide basic information for the comprehensive evaluation of cancer patients who received regular treatment from a tumor hospital in a rural area of China, and also to provide a functional approach for the application of PBCR follow-up in the HBCR.

\section{Conclusions}

Our study highlights the survival from a special patient population who were treated / registered in a hospital-based cancer registry, but whose survival status was identified by both active and passive follow up through well-run population-based cancer registries as well. The survival rates of each site or of all sites-combined in this setting are consistent with those described elsewhere in China and abroad. Discrepancies in overall survival could be affected by the weight (or proportion) of sites with or without better prognosis. The hospital-based cancer survival is a better index to evaluate the outcomes that reflects the levels of comprehensive treatment and improvement of medical and health services.

\section{Abbreviations}

HBCR, hospital-based cancer registry; PBCR, population-based cancer registry; OS, observed survival; RS, relative survival; SE, standard error; NHL, non-Hodgkins lymphoma; CNS, central nervous system.

\section{Acknowledgements}

We thank the Qidong Cancer Registry and the Haimen Cancer Registry for their contribution in providing cancer database for checking and follow-up. We thank Prof. Thomas W. Kensler of Johns Hopkins Bloomberg School of Public Health and University of Pittsburgh, USA for his helpful comments and language editing. This work was supported by the Nantong Bureau of Science and Technology (QYZ15017, CP22012005).

\section{Competing Interests}

The authors have declared that no competing interest exists.

\section{References}

1. Chen W, Zheng R, Baade PD, et al. Cancer statistics in China, 2015. CA Cancer J Clin. 2016; 66(2): 115-132.

2. Chen JG, ed. Cancer in Qidong, 1972-2011. Beijing: Military Medical Science Press; 2013: 1-346.

3. Wang HS, Huang PX, Tang JG, et al. Incidence and trend of malignancies from 2001 to 2007 in Haimen City, Jiangsu Province. China Cancer. 2010; 19(2): 100-104.

4. Zeng H, Zheng R, Guo Y, et al. Cancer survival in China, 2003-2005: a population-based study. Int J Cancer. 2015; 136(8): 1921-1930.

5. Chen JG, Zhu J, Zhang YH, et al. Cancer survival in Qidong, China, 1992-2000. In: Sankaranarayanan R, Swaminathan R, eds. Cancer Survival in Africa, Asia, the Caribbean and Central America. IARC Sci Pub No. 162. Lyon: IARC; 2011: 43-53.

6. Chen JG, Zhu J, Zhang YH, et al. Cancer survival in Qidong between 1972 and 2011: A population-based analysis. Mol Clin Oncol. 2017; 6(6): 944-954.

7. Hong HY, Pei XF, Li CY, et al. 5 Years Survival Follow-up of 154 cases of nasopharyngeal carcinoma in Zhuhai. J Sun Yat-Sen Univ (Med Sci). 2012; 33(1): 136-140

8. Cao X, Wen ZS, Wang XD, et al. The clinical effect of metformin on the survival of lung cancer patients with diabetes: A comprehensive systematic review and meta-analysis of retrospective studies. J Cancer. 2017; 8(13): 2532-2541.

9. Chen HZ, Chen JG, Zhang LF, et al. Current status and progress of cancer follow-up. Chin J Dis Ctrl Prev. 2015; 19(5): 517-523.

10. Chen HZ, Yue ZJ, Chen JG, et al. Analysis on characteristics of inpatients with cancers in Nantong Tumor Hospital. Chin J Cancer Prev Treat. 2015; 22(12): 901-906.

11. Wei KR, Chen WQ, Zhang SW, et al. Cancer registration in the Peoples Republic of China. Asian Pac J Cancer Prev. 2012; 13: 4209-4214.

12. National Health and Family Planning Commission of the PRC, State Bureau of Traditional Chinese Medicine. Notice on Printing and Distributing the Measures for the Administration of Cancer Registration ([2015] No. 6). The Measures for the Administration of Cancer Registration. Revised 23 September 2017; http://www.gov.cn/gongbao/content/2015/content_28688 79.htm

13. Forman D, Bray F, Brewster DH, eds. Cancer Incidence in Five Continents Volume X. IARC Sci Publ No. 164. Lyon: IARC; 2014: 1-1365.

14. Chen YS, Chen JG, Zhu J, et al. Long-term survival trends of gastric cancer patients between 1972 and 2011 in Qidong. Chin J Cancer. 2015; 34(12): 602-607.

15. Yu LY, Liu L, Mu HJ, et al. Analysis on bias in cancer survival estimation from population-based passive follow up study. Chin J Cancer Prev Treat. 2015; 22(6): 407-410.15.

16. Investigation Group of Action Plan for Cancer Prevention and Control in China. Spot investigation report of action plan for cancer prevention and control in China. China Cancer. 2012; 21(9): 641-643.

17. Xiang YB. Statistical analysis for data of tumor epidemiological studies: Lecture 5, estimation of relative survival rate. Chin J Epidemiol. 1999; 20(2): 55-57.

18. Parkin DM, Hakulinen T. Chapter 12. Analysis of survival. In: Jensen OM, Parkin MD, MacLennan R, eds. Cancer Registration: Principles and Methods. IARC Sci Publ No. 95. Lyon: IARC; 1991: 159-176.

19. Miller KD, Siegel RL, Lin CC, et al. Cancer treatment and survivorship statistics, 2016. CA Cancer J Clin. 2016; 66(4):271- 289.

20. Tanaka H, Tanaka M, Chen W, et al. Proposal for a cooperative study on population-based cancer survival in selected registries in East Asia. Asian Pac J Cancer Prev. 2009; 10(6): 1191-1198. 
21. Yeole BB, Kumar AV. Population-based survival from cancers having a poor prognosis in Mumbai (Bombay), India. Asian Pac J Cancer Prev. 2004; 5(2): 175-812.

22. Pathy NB, Yip $\mathrm{CH}$, Taib NA, et al. Breast cancer in a multi-ethnic Asian setting: results from the Singapore-Malaysia hospital-based breast cancer registry. Breast. 2011; 20 Suppl 2:S75-80.

23. Fang $\mathrm{YJ}, \mathrm{Wu} \mathrm{XJ}$, Zhao Q et al. Hospital-based colorectal cancer survival trend of different tumor locations from 1960s to 2000s. PLoS One. 2013; 8(9): e73528.

24. Cheng $\mathrm{HY}$, Chu $\mathrm{CH}$, Chang $\mathrm{WH}$, et al. Clinical analysis of multiple primary malignancies in the digestive system: a hospital-based study. World J Gastroenterol. 2005; 11(27):4215-4219.

25. Zuo T, Zheng H, Li H, et al. The influence of stage at diagnosis and molecular subtype on breast cancer patient survival: a hospital-based multi-center study. Chin J Cancer. 2017; 36: 84. Published online 2017 Oct 25. doi: 10.1186/s40880-017-0250-3.

26. Higashi T, Nakamura F, Shibata A, et al. The national database of hospital-based cancer registries: a nationwide infrastructure to support evidence-based cancer care and cancer control policy in Japan. Jpn J Clin Oncol. 2014; 44(1): 2-8.

27. Tamminga SJ, Verbeek JHAM, Bos MMEM, et al. Effectiveness of a hospital-based work support intervention for female cancer patients - a multi-centre randomised controlled trial. PLoS ONE. 2013; 8(5): e63271. 